\title{
APPENDIX
}

\section{Challenging Tube and Slip-Link Models: Predicting the Linear Rheology of Blends of}

\section{Well-Characterized Star and Linear 1,4-Polybutadienes}

Priyanka S. Desai, ${ }^{*}+$ Beom-Goo Kang, ${ }^{+} \neq$Maria Katzarova, ${ }^{\&} \neq$ Ryan Hall, ${ }^{*}$ Qifan Huang, ${ }^{*}$ Sanghoon Lee, ${ }^{\S}$ Maksim Shivokhin, ${ }^{\&}$ Taihyun Chang, ${ }^{\S}$ David C. Venerus, ${ }^{\&}$ Jimmy Mays, ${ }^{+}$Jay D. Schieber, ${ }^{\&, \$}$ Ronald G. Larson*\#,@

Here we consider the ability of alternative versions of the tube model to predict the rheology of the four sets of star/linear blends available in literature, namely, the data set from the main draft (1,4-PBd 24KS-58KL), data set from Struglinski et al. ${ }^{12}$ which was also studied by Milner et $a l^{5}{ }^{5}(1,4-\mathrm{PBd} 42.5 \mathrm{KS}-105 \mathrm{KL})$, data set from Shivokhin et al. ${ }^{14}$ (1,4-PBd 27.4KS6.9KL), and data set from Roovers ${ }^{16}$ (1,4-PBd 342KS-23.6KL).

We also present additional CFSM slip-link simulation calculations for blends 1,4-PBd 24KS-58KL and the blends from Shivokhin et al. ${ }^{14}, 1,4-\mathrm{PBd} 27.4 \mathrm{KS}-6.9 \mathrm{KL}$.

\section{Different versions of tube model predictions for data set from the main text $(1,4-P B d$} 24KS-58KL)

1.i. Calculations using different arm fluctuation options during supertube relaxation: fat, skinny tubes, or "arm frozen."

According to common versions of the tube theory, after the linear chains relax, the remaining un-relaxed star arms explore a widening tube called the "supertube" by constraintrelease Rouse (CR-Rouse) motion. The Hierarchical model allows different options for the retraction of the star arm in the CR-Rouse regime. The arm can undergo retraction in the thin tube whose diameter is set by the un-relaxed volume fraction $\phi$ of all chains, including the linear 
ones, just before the onset of CR-Rouse, relaxation. Alternatively, arm retraction can take place in the fat tube (or supertube) whose diameter is set by $\phi_{S T}$ and is continuously evolving within the CR-Rouse regime. Or the arm can be assumed not to retract at all during period of supertube exploration, which is the so-called "arm frozen" assumption initially invoked by Milner et al. ${ }^{5}$. Figure A1 shows experimental G' data for the blend $24 \mathrm{KS}-58 \mathrm{KL}$ which is studied in the main text and lines show Hierarchical model predictions done using Park parameters. In Figure A1, the model over-predicts the data if the star arm is allowed to relax within the thin tube (solid lines). However, when the star arm is allowed to relax in the fat tube (dashed lines), the modulus decreases rather quickly and is closer to the experimental data, although still not matching the data well. Figure A1-B shows increasingly poorer predictions as the star volume fraction decreases when the arm is 'frozen' or not allowed to retract at all during the CR-Rouse regime. The results in Figure A1-B show that while the ad hoc "arm frozen" assumption seems to have yielded good predictions for the set of star/linear blends studied by Milner et al. ${ }^{5}$, it does not provide consistently accurate predictions. 

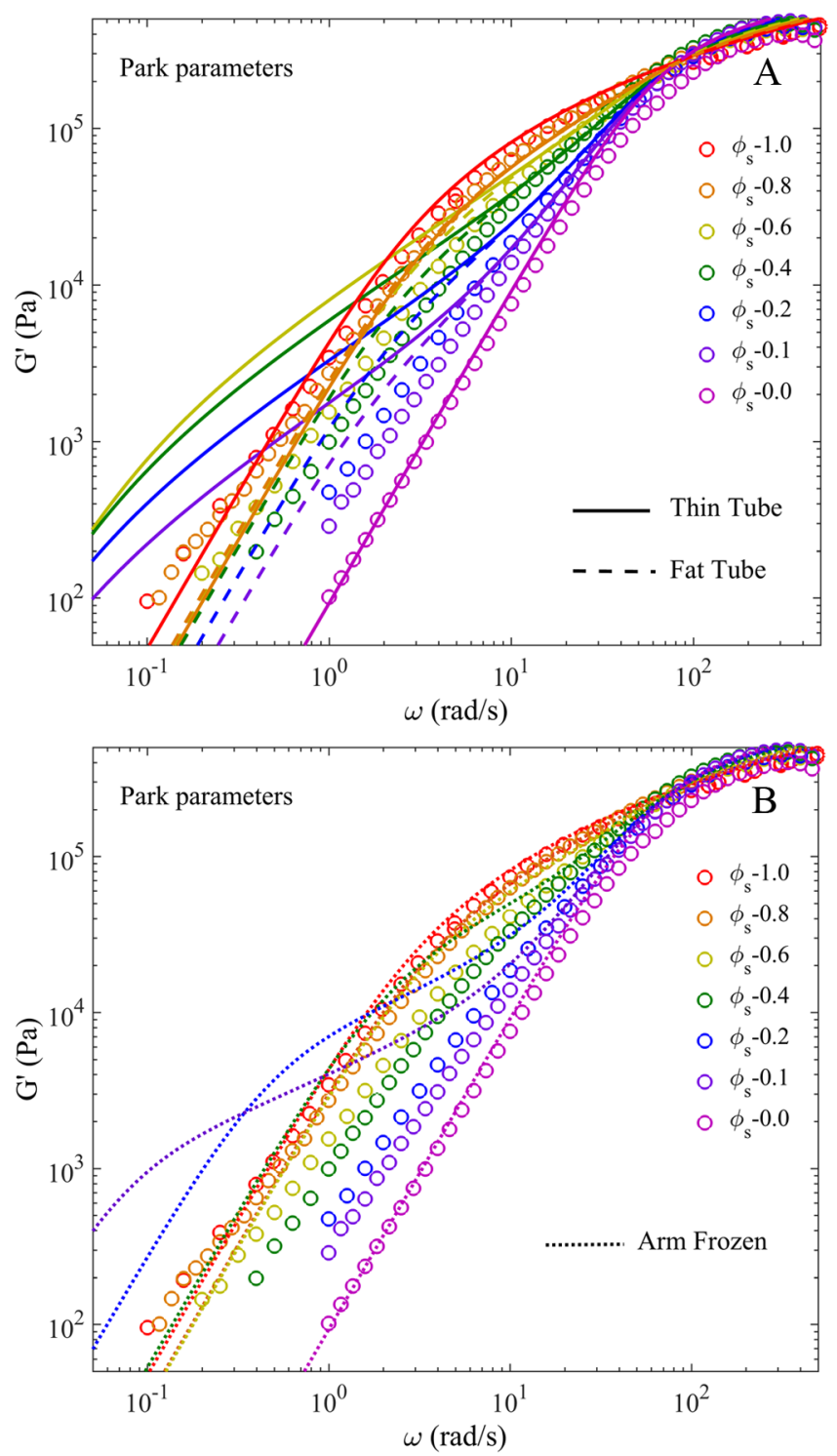

Figure A1. Storage moduli, G' for 1,4-PBd 24KS-58KL blends with decreasing fraction of starbranched chains from left to right as shown. The symbols are experimental data at $\mathrm{T}=25^{\circ} \mathrm{C}$. In A, the dashed lines show predictions for arm-retraction in the fat tube, solid lines show arm retraction in thin tube while in B, the dotted lines show predictions with the 'arm frozen' algorithm in the CR-Rouse regime, using Park parameters.

1.ii. Calculations using different parameter sets within the tube model: Park versus Das parameters 

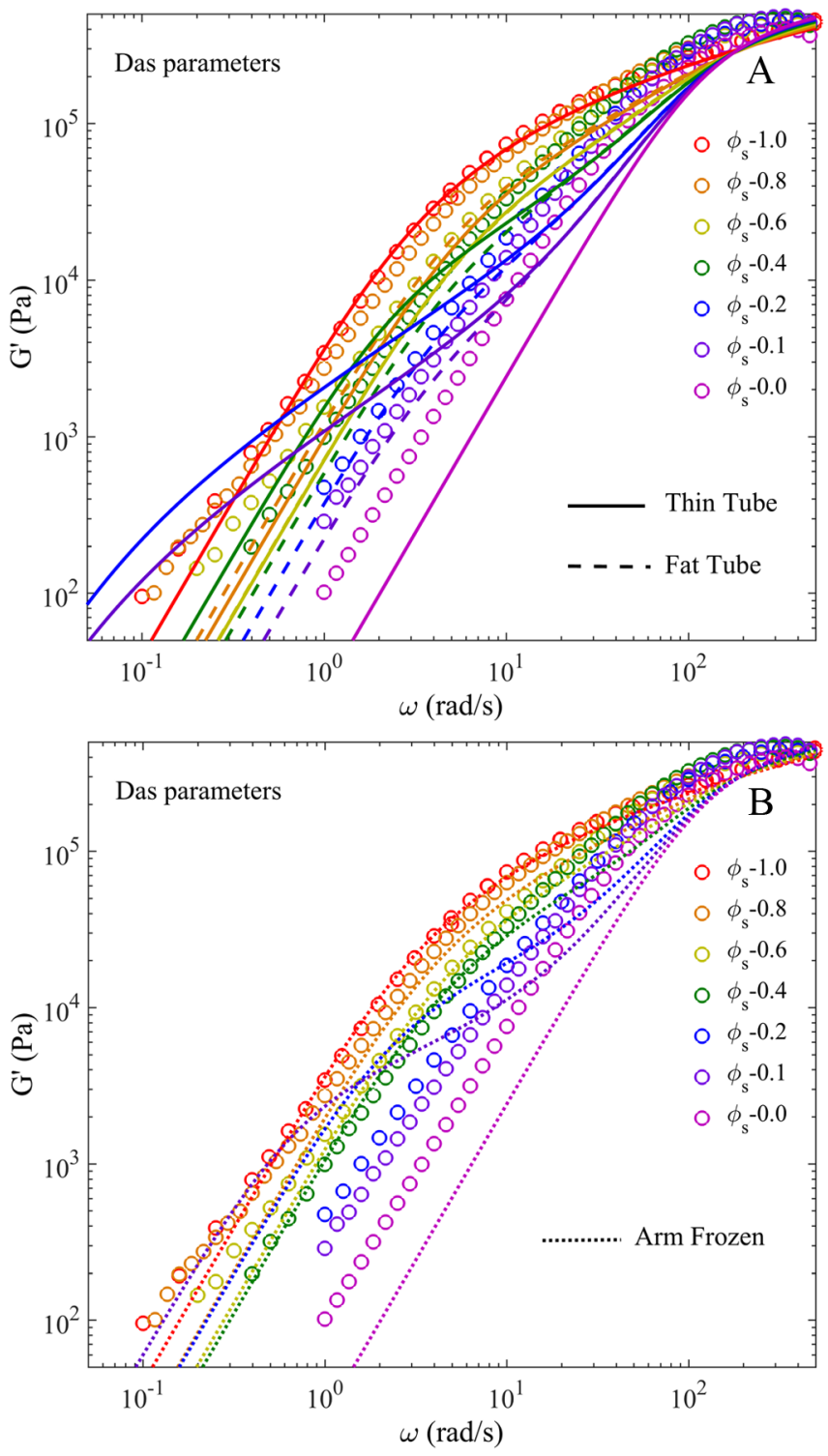

Figure A2. Same as Figure A1, except that the predictions are done using Das parameters in both $\mathrm{A}$ and $\mathrm{B}$.

Figure A2 shows the same calculations as in Figure A1, except the predictions use the 'Das' $(\alpha=1)$ set of parameters. In both Figure A2-A and A2-B, using Das parameters underestimates the modulus of the pure linear polymer, 58KL. Similar to what is seen in Figure A1, the model overestimates the experimental data during thin tube arm retraction as shown by 
solid lines in Figure A2-A and gets closer to the experiments if arm retraction is assumed in fat tube (dashed lines in figure A2-A). In general, the fat tube assumption predicts faster relaxation than does retraction in thin tube. In Figure A2-B, where the arm frozen option is used during the CR-Rouse regime, the predictions worsen relative to the fat tube predictions, particularly for cases with low star volume fractions, just as they do when the Park parameters are used.

Note that even though both the Park and Das parameters have somewhat different input values for the plateau modulus $G_{N}^{0}$, the entanglement spacing $M_{e}$, and the equilibration time $\tau_{e}$, the most notable difference between the two sets is the value of the dilution exponent, $\alpha$; i.e., $\alpha=$ 1 for Das parameters while $\alpha=4 / 3$ for Park parameters. Comparing Figures A1 and A2, one would notice that regardless of the CR-Rouse arm retraction algorithm used, Das parameters $(\alpha$ =1) are able to give predictions closer to the experimental data for all of different star volume fractions in blends of $24 \mathrm{KS}-58 \mathrm{KL}$ as compared the predictions done using Park parameters. However, Park parameters result in an excellent fit to the data for the pure star and linear components, which is not achieved with Das parameters.

1.iii. Calculations including the disentanglement relaxation mechanism

Examination of supertube relaxation: The density of the remaining entanglements on the unrelaxed star arms after the linear chains have relaxed varies as a function of the star volume fraction. More specifically, the remaining number of entanglements are obtained by dividing the molecular weight of the star arm, $M_{a}$, by the entanglement molecular weight, $M_{e}$, and multiplying the resulting value by the total un-relaxed volume fraction, $\phi$. Figure A3 shows the unrelaxed volume fraction, $\phi$ and supertube volume fraction, $\phi_{S T}$ as a function of time for PBd 
24KS-58KL blends at various star volume fractions. For all the cases shown in Figure A3, the linear chains reach their reptation time at around $t=0.0145 \mathrm{~s}$, at which time they completely relax. For the case where the star volume fraction is relatively high, for example, $\phi_{s}=0.8$ as shown in Figure A3-B, the total un-relaxed volume fraction $\phi$ suddenly drops at $\mathrm{t}=0.0145 \mathrm{~s}$ from 0.536 to 0.417 , a drop of only about $22 \%$. For this case, since the density of remaining entanglements per arm is around 6, which is still considerable, the star arm is still sufficiently entangled that $\phi_{S T}$ is able to catch up to $\phi$ in a reasonably short time during the CR-Rouse regime. Consequently, the storage modulus G' for this blend in Figure 6A (in the main text) does not relax slower than the pure star does. However, if the star volume fraction is low, for example, $\phi_{s}=0.1$ as shown in Figure A3-E, the sudden drop at $\mathrm{t}=0.015 \mathrm{~s}$ in the total un-relaxed volume fraction $\phi$ is much more appreciable, from a value of 0.591 down to only 0.053 . The corresponding density of entanglements abruptly drops by about $91 \%$ and there are only about 0.8 surviving long-time entanglements on each star arm. For this low number of surviving entanglements, $\phi_{S T}$ never drops down to $\phi$ and this leads to a prolonged supertube regime. Consequently, the modulus relaxes very slowly as seen in Figure 6A (in the main text). A similar trend is observed in Figure A3-D, for star volume fraction, $\phi_{s}=0.2$. 

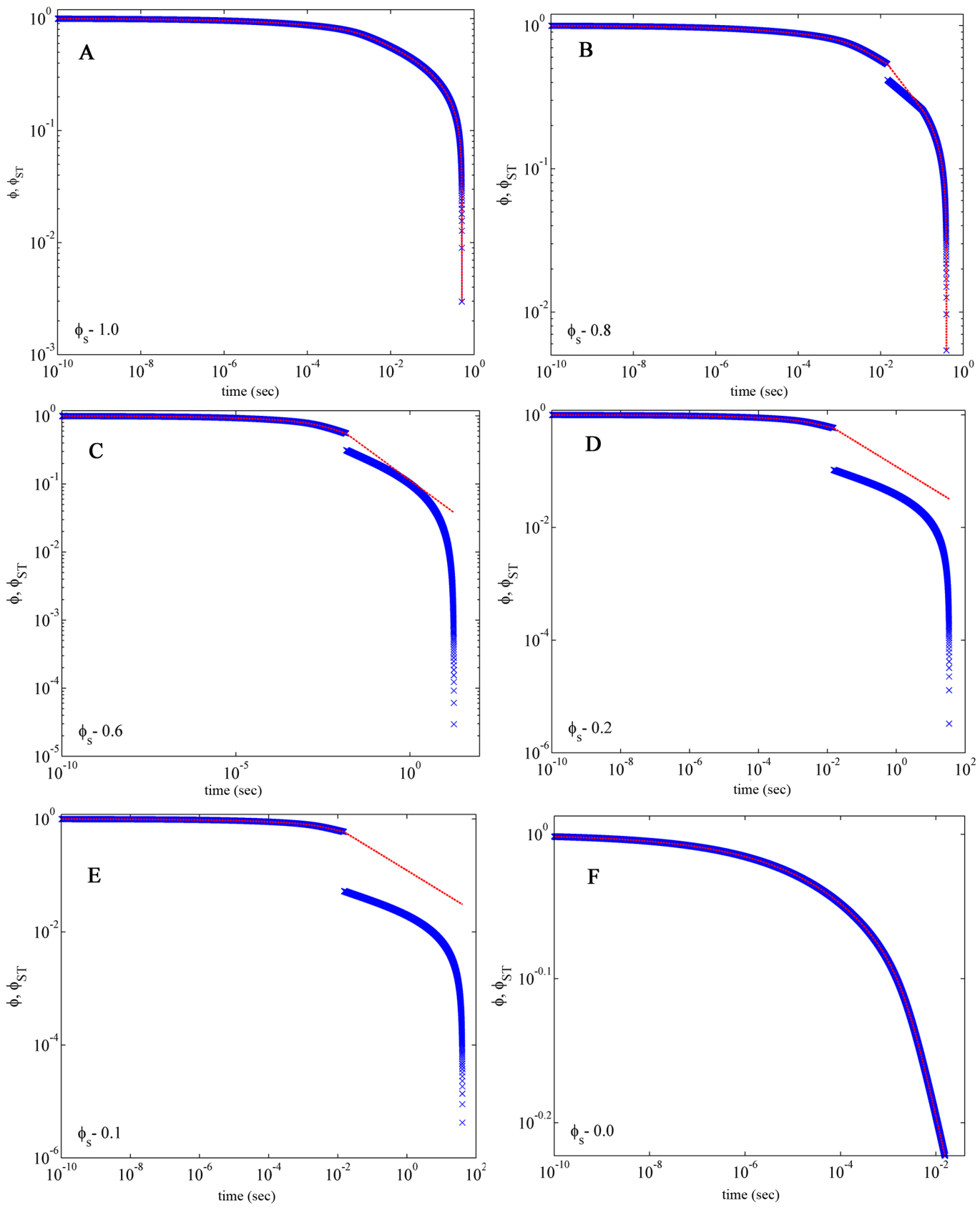

Figure A3. Hierarchical model predictions (with parameters described in the caption to Figure 6 (in the main text) for un-relaxed volume fraction $\phi$ (blue symbols) and supertube volume fraction $\phi_{S T}$ (red dotted lines) as a function of time for 1,4-PBd 24KS-58KL blends with star volume fractions $\phi_{s}-1.0,0.8,0.6,0.2,0.1,0.0$ (A, B, C, D, E, F respectively). 
Disentanglement relaxation: The above failure of the tube model is related to its inability to model constraint release properly as a combination of CR-Rouse relaxation and tube dilation. For star-linear blends, if the volume fraction of stars is lower than around $20 \%$, both the Hierarchical and $\mathrm{BoB}$ models, using the "thin tube" assumption generally predict stress relaxation that is much slower than that observed in experiments. The reason is that that once the short linear chains are relaxed by reptation, there is a sudden drop of the un-relaxed volume fraction $\phi$ (by about $90 \%$ ) and the system enters the "supertube" relaxation regime in which the star relaxes by the CR-Rouse motion of the thin confining tube, and $\phi_{S T}$ follows a power law $\phi_{S T} \propto t^{-1 / 2 \alpha}$, where $\alpha$ is the "dilution exponent." Only when $\phi_{S T}$ drops below $\phi$ is the CR-Rouse relaxation process deactivated. Since for small star volume fractions, $\phi$ is very low when the linear chains relax, it takes an extremely long time for $\phi_{S T}$ to drop down to $\phi$, which leads to an artificially prolonged supertube regime, as shown in Figure A3. In an attempt to "fix" these model predictions, Larson ${ }^{7}$ deployed a "disentanglement relaxation threshold" that allows the star arm to completely relax when the tube in which it fluctuates has dilated to the point that it has only $S_{a, \min }=1$ to 3 remaining entanglements, which is an entanglement density at which a transition to strong entanglement effects typically occurs. A discussion of this disentanglement relaxation mechanism can be found in Wang et al. ${ }^{9}$ For consistency, this disentanglement threshold should be applied also to the linear components of the blend.

In the blends studied here, namely the PBd24KS-58KL blends, the linear chains are quite short and for the case shown in Figure A4-C where the star volume fractions are very low, the linear chains make up the majority of the system. To include the disentanglement relaxation, here we assume that once the linear chains reach its disentanglement threshold of $S_{a, \min }=3$ 
entanglements, the chains are allowed to relax their stress even before they reach their reptation time. Once the linear chains are relaxed, they dilate the tube containing the star arms, allowing them to retract faster and quickly reach their disentanglement relaxation threshold of 3 entanglements and also relax. This leads to G' predictions for $S_{a, \min }=3$ that are even lower than the experiments, for the four cases considered here in Figure A4-C. This might be corrected by either applying the disentanglement mechanism only to the star polymers, or by changing the value of the threshold to a lower value, $S_{a, \min }=1$ as done by Larson ${ }^{7}$ and shown here in Figure A4-A and $S_{a, \min }=2$ in A5-B. Of these three values, $S_{a, \min }=2$ works best for PBd24KS-58KL blends. 

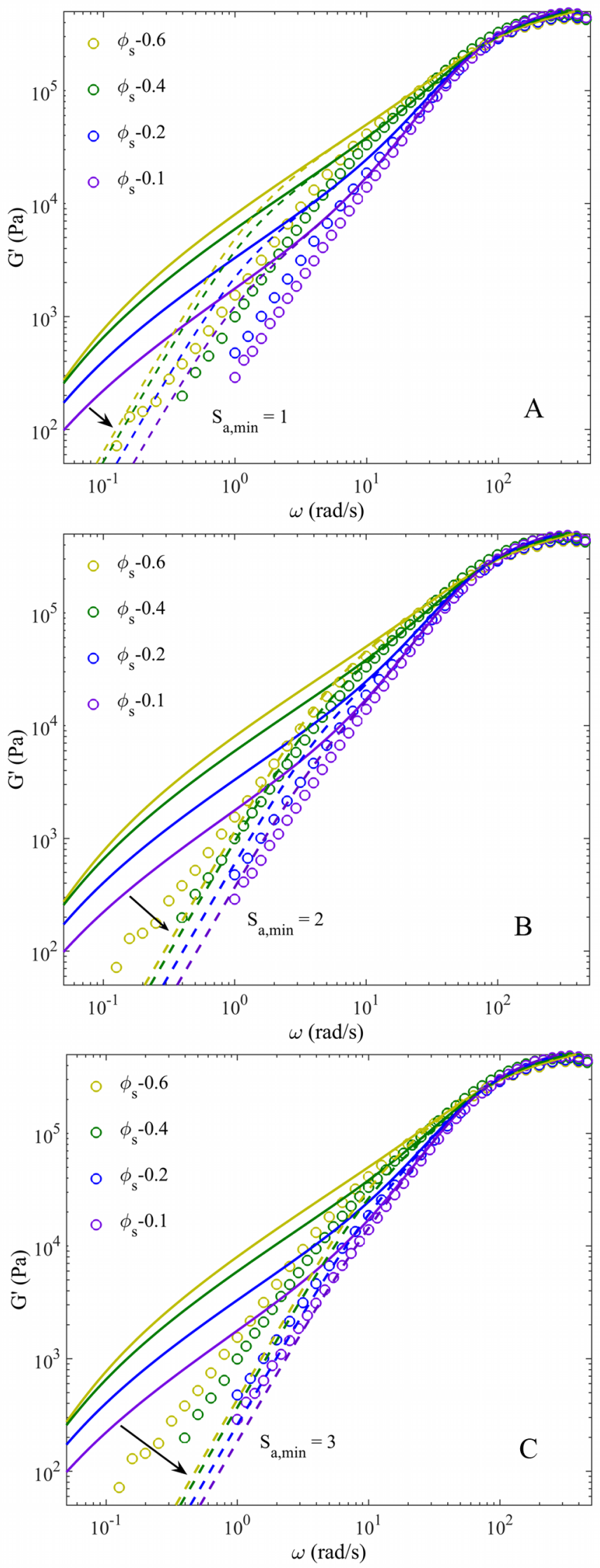
Figure A4. Data (symbols) and predictions for arm-retraction using Park input parameters in a "thin" tube (solid lines) taken from Fig. 5A (in the main text) for four blends, with dashed lines giving the predictions with disentanglement relaxation process activated at the entanglement threshold value of A: $S_{a, \min }=1$, B: $S_{a, \min }=2$ and C: $S_{a, \min }=3$.

1.iv. Calculations using different algorithms within the tube model framework: Hierarchical model, BoB model and the special star-linear algorithm developed by Park and Larson ${ }^{57}$

To investigate a different version of the tube model, the BoB model was tested using the Das parameters normally used for this model. As can be seen in Figure A5, the BoB model predicts the rheology of blends with star fractions $\phi_{s} \geq 0.6$ better than does the Hierarchical model, but predictions for all other blends are just as poor. This failure in Figure A5 for blends with $\phi_{s} \leq 0.2$ and in Figure 6 (in the main text) for blends with $\phi_{s} \leq 0.6$ is again due to the prolonged "supertube" relaxation region discussed in the next section. Interestingly, unlike the Hierarchical model, the BoB model is unable to predict the pure linear 58KL correctly.

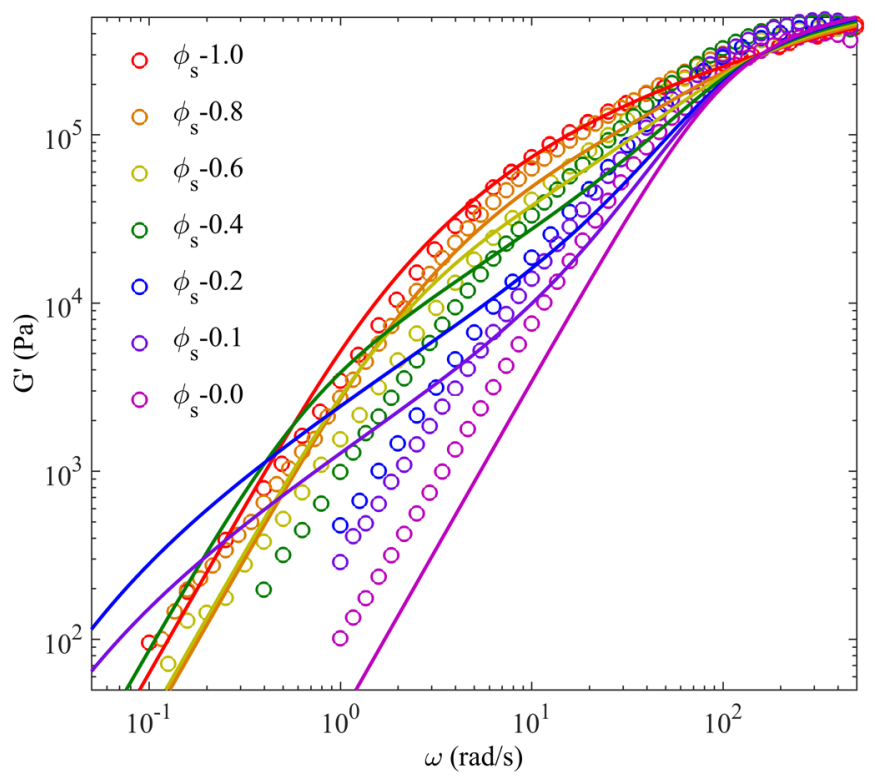


Figure A5. Same as Figure A1, except that the solid lines are BoB tube model predictions using the 'Das' input parameters and arm retraction in thin tube during the CR-Rouse regime.

Park and $\operatorname{Larson}^{57}$ have also developed a specific algorithm to predict linear viscoelasticity of star and linear bidisperse blends which is based on the theory and assumptions of the Milner-McLeish model developed in Milner $e t ~ a l .{ }^{5}$ including early-time fluctuations, latetime retractions, and constraint-release Rouse motion for star/linear blends. This algorithm assumes the arm retraction to be frozen during the CR-Rouse motion, according to the MilnerMcLeish theory. In their paper, they tested the theory using two sets of parameters, the first of which we refer to here and in the main text as the "Park" input parameters $\left(\alpha=4 / 3, M_{e}=1650\right.$, $G_{N}^{0}=1.15 \mathrm{MPa}, \tau_{e}=3.5 \times 10^{-7} \mathrm{~s}$ at $\left.\mathrm{T}=25^{\circ} \mathrm{C}\right)$ and the second of which we here call the "Park ( $\alpha=1)$ " input parameters $\left(\alpha=1, M_{e}=2200, G_{N}^{0}=1.15 \mathrm{MPa}, \tau_{e}=9.5 \times 10^{-7} \mathrm{~s}\right.$ at $\left.\mathrm{T}=25^{\circ} \mathrm{C}\right)$. We have tested the special star/linear algorithm using both the "Park" and and "Park $(\alpha=1)$ " input parameters.

As can be seen in Figures A6 and A7, the special star-linear algorithm predicts the rheology of blends better only when disentanglement relaxation at a threshold of $S_{a, \min }=2$ is included. Furthermore, the predictions with the "Park" set of parameters, as shown in Figure A6 closely resemble the experimental data than do the predictions done with "Park $(\alpha=1)$ " input parameters as shown in Figure A7. 


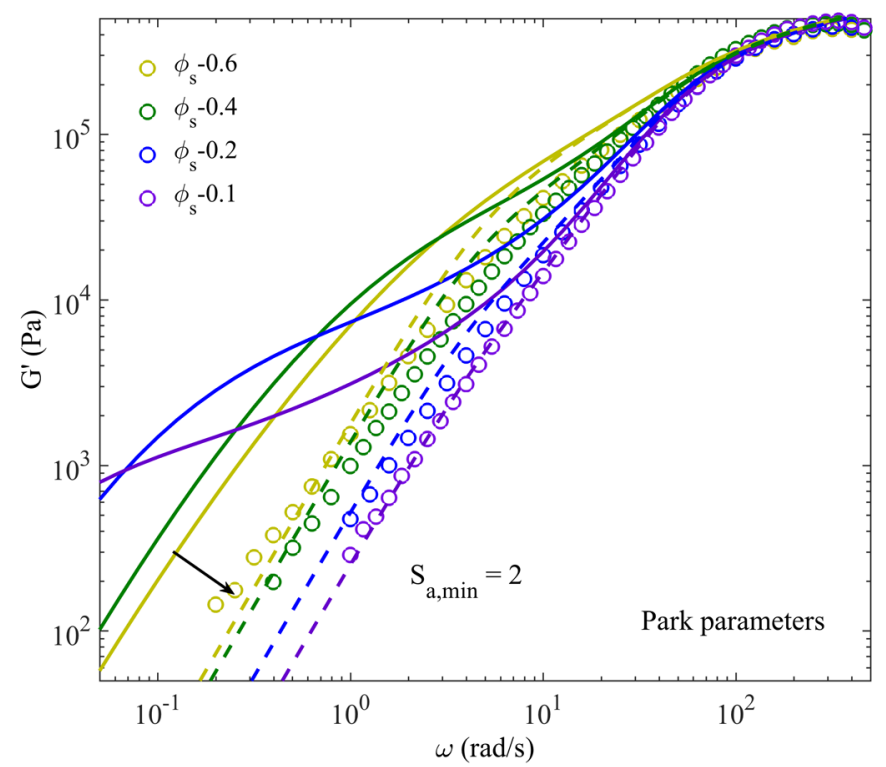

Figure A6: Data are same as Figure A4, and the predictions are for the special star-linear algorithm model using the "Park" input parameters from Park and Larson" ${ }^{57}$. Solid lines show predictions without the disentanglement process activated. Dashed lines give predictions with disentanglement relaxation process activated at the entanglement threshold value of $S_{a, \min }=2$.

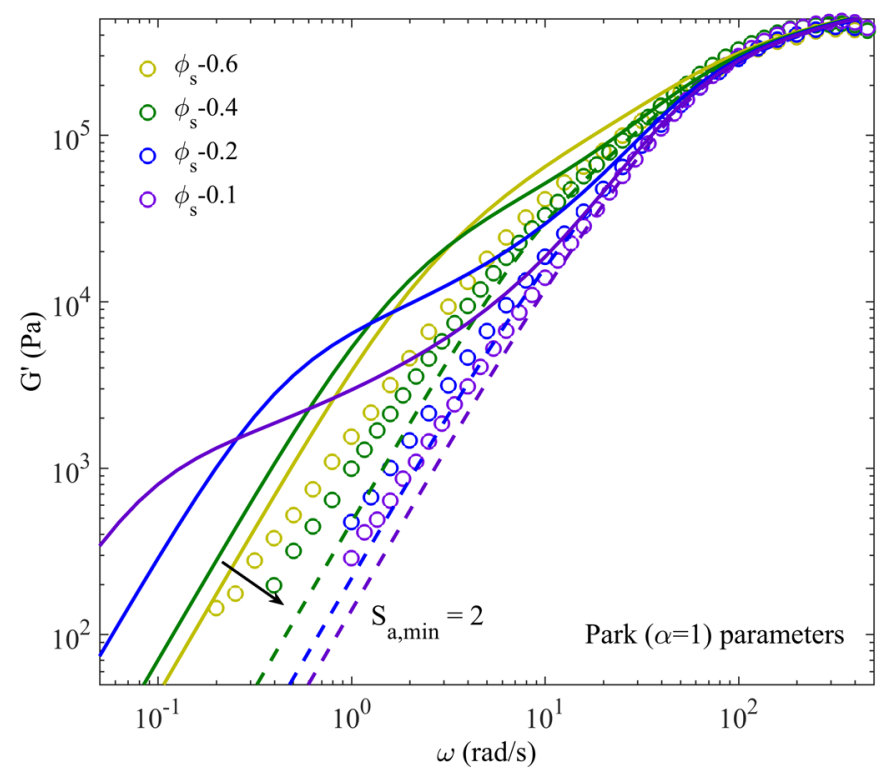


Figure A7: Data are same as Figure A4, and the predictions are for the special star-linear algorithm model from Park and $\operatorname{Larson}^{57}$ using the "Park $(\alpha=1)$ " input parameters. Solid lines show predictions without the disentanglement process activated. Dashed lines give predictions with disentanglement relaxation process activated at the entanglement threshold value of $S_{a, \min }=$ 2.

\section{Different versions of tube model predictions for the classical data set from Struglinski $e t$ $a l .{ }^{13}$ (also reported in Milner et al..$\left.^{5}\right)(1,4-\mathrm{PBd} 42.5 \mathrm{KS}-105 \mathrm{KL})$}

2.i. Calculations using different arm fluctuation options during supertube relaxation: fat, skinny tubes, or "arm frozen."

Figure A8 presents the Hierarchical model predictions using Park parameters for the storage modulus, G', of the PBd 42.5KS-105KL blends, using the three different arm-retraction algorithms as described in the main text and as done in Figure A1. Similar to the trend observed in Figure A1, the no-arm-retraction algorithm in general predicts slower relaxation in the terminal region than the two other options because by preventing arm retraction, it imposes a constant and thus higher value of the unrelaxed volume fraction, $\phi(t)$ in the CR-Rouse regime. Arm retraction in either the thin or the fat tube leads to a faster decay of $\phi(t)$ as compared to noarm retraction but it also might lead to a longer CR-Rouse regime as a result of the longer time required for the supertube volume fraction, $\phi_{S T}$ to meet with $\phi(t)$. Notably, the arm-frozen algorithm applied in Figure A8-B gives the closest predictions to the experimental data as shown except for the case of the blend with a low star volume fraction, $\phi_{s}-0.2$. This is because of the wider CR-Rouse regime and a longer time required for $\phi_{S T}$ to catch-up to $\phi(t)$. 

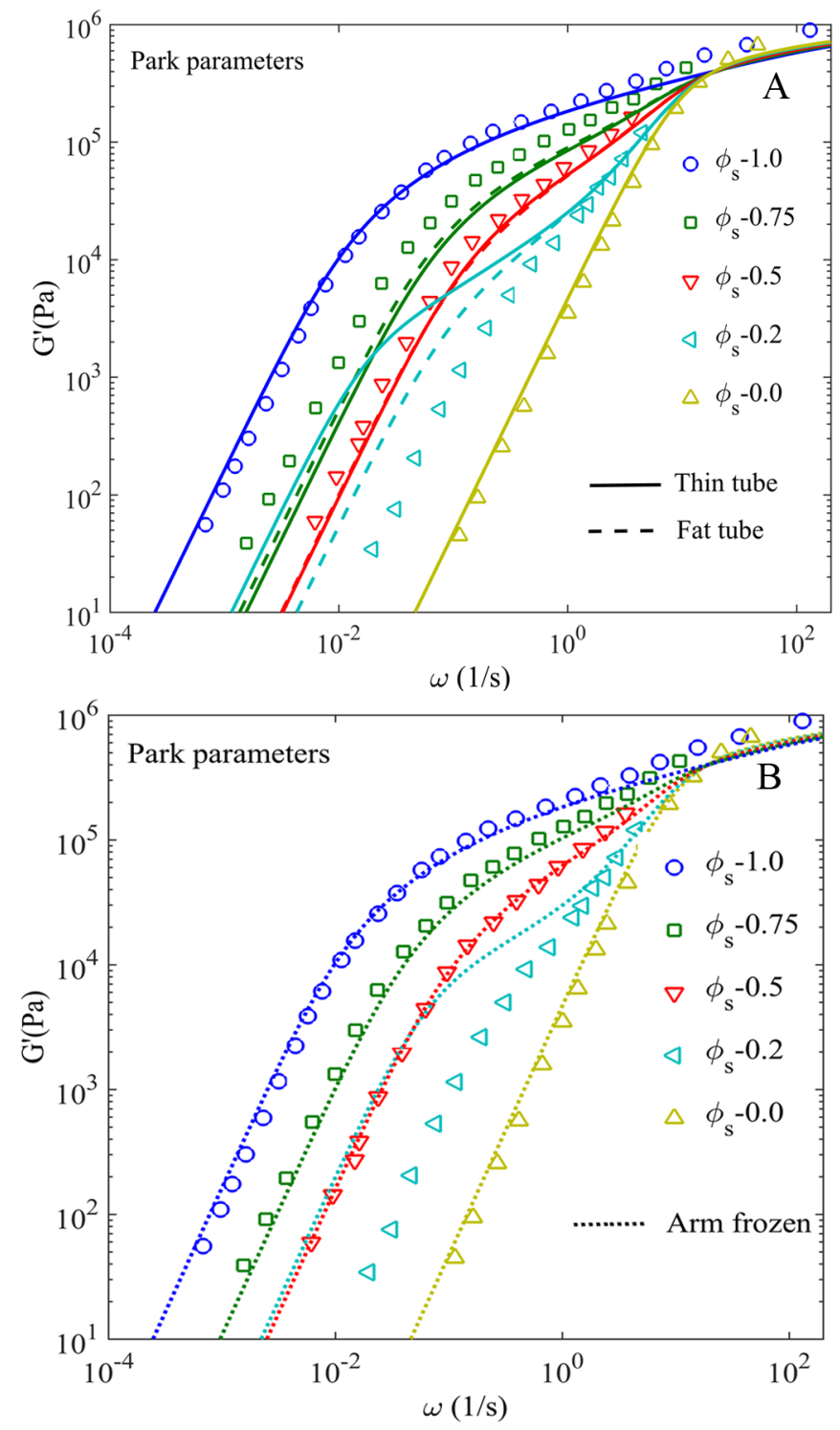

Figure A8. Storage moduli, G' for 1,4-PBd 42.5KS-105KL blends (data of Struglinski et al. cited in the Milner-McLeish paper, Ref. 5) at five different star volume fractions as shown. The symbols are experimental data at $\mathrm{T}=25^{\circ} \mathrm{C}$. In $\mathrm{A}$, the dashed lines show predictions for armretraction in the fat tube, solid lines show arm retraction in thin tube while in $\mathrm{B}$, and the dotted lines show predictions with the 'arm frozen' algorithm in the CR-Rouse regime, using Park parameters. 
Figure A9 shows the Hierarchical model predictions for the storage modulus, G', of the PBd 42.5KS-105KL blends, using the three different arm-retraction algorithms using Das parameters. As observed in Figure A2 for PBd 24KS-58KL, for PBd 42.5KS-105KL the model also mostly fails to predict the pure linear and pure star rheology in either of the arm-retraction algorithms, although the arm-frozen algorithm shown in Figure A9-B yields predictions reasonably close to the data while missing both pure star and linear components. Note that the "arm-frozen" assumption with Das parameters gives very poor predictions for the PBd 24KS-58KL blends, as shown in Fig. A2-B. Interestingly, these were the same set of assumptions $(\alpha=1$ and arm-frozen during CR-Rouse regime) employed by Milner et $a .^{5}$ for the same data set reported in their paper, and they obtained good agreement with the data for PBd $42.5 \mathrm{KS}-105 \mathrm{KL}$ at all volume fractions. However, the Hierarchical model uses a slightly different numerical approach than the MM theory in Milner et al. ${ }^{5}$ albeit using the same analytical formulas developed in the MilnerMcLeish theory to calculate the arm retraction potential $U_{\text {eff }}$ and the relaxation time, $\tau_{\text {late }}$. Thus, while the Hierarchical and BoB models contain the same basic physics as that of the model of Milner et $a l .{ }^{5}$, and give similar predictions as the Hierarchical and BoB models for pure linear and pure star polymers (when parameters are the same), predictions for star/linear blends are apparently extremely sensitive to the algorithmic implementation of these physics. 

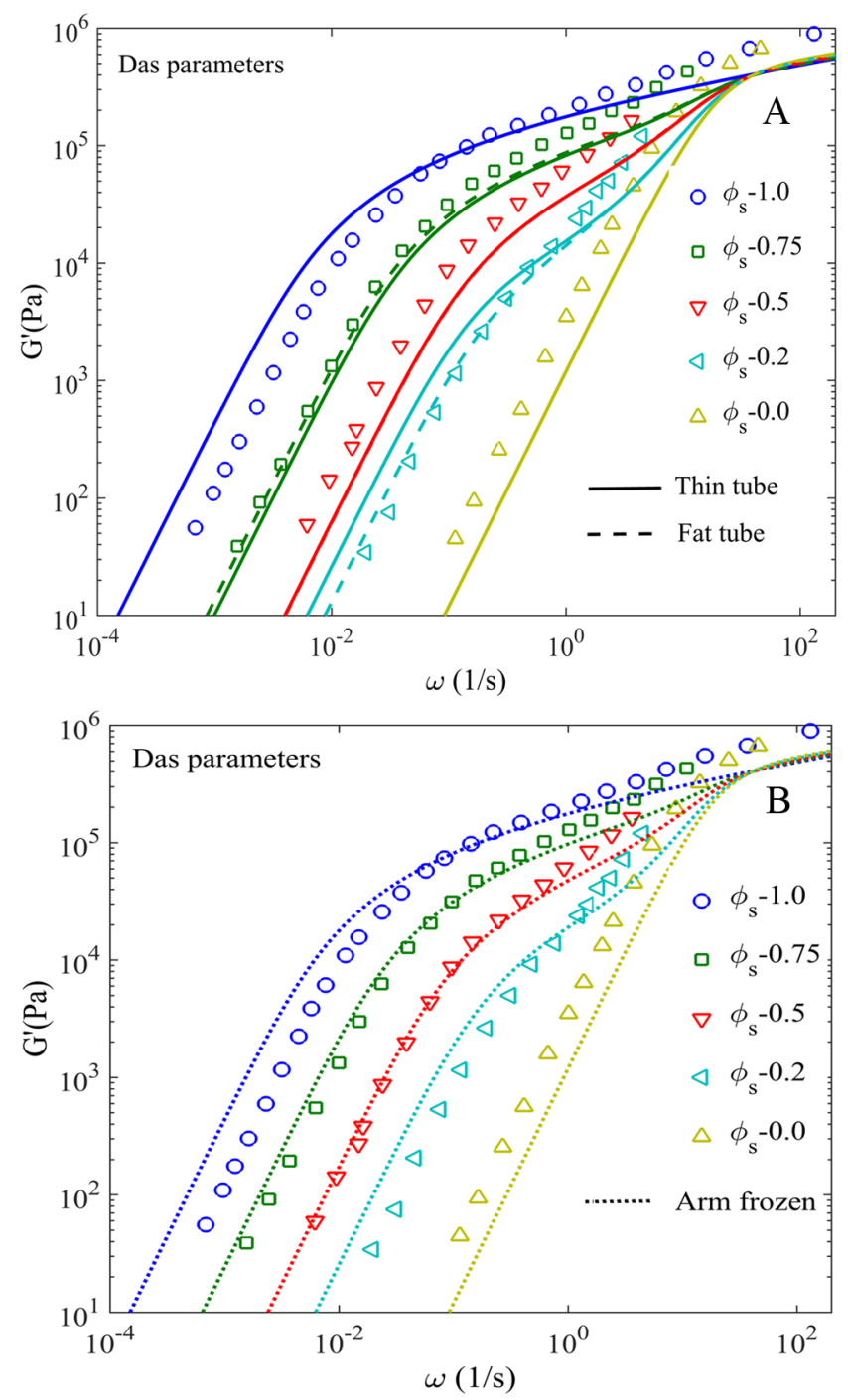

Figure A9. Same as Figure A8, except using Das parameters.

2.ii. Calculations including the disentanglement relaxation mechanism

In order to cut-off the artificially extended CR-Rouse regime, disentanglement relaxation mechanism is switched ON with different relaxation thresholds in Figure A10 for the case of a low star volume fraction, $\phi_{s}=0.2$ in the blend $1,4-\mathrm{PBd} 42.5 \mathrm{KS}-105 \mathrm{KL}$. As shown, using $S_{a, \min }$ value of 3 provides the best fit to the experimental data, while a threshold of $S_{a, \min }=1$ or $S_{a, \min }=$ 2 overestimates the data. Note that using disentanglement relaxation for blends with higher star 
volume fractions underestimates the experimental data of 1,4-PBd 42.5KS-105KL (not shown here).

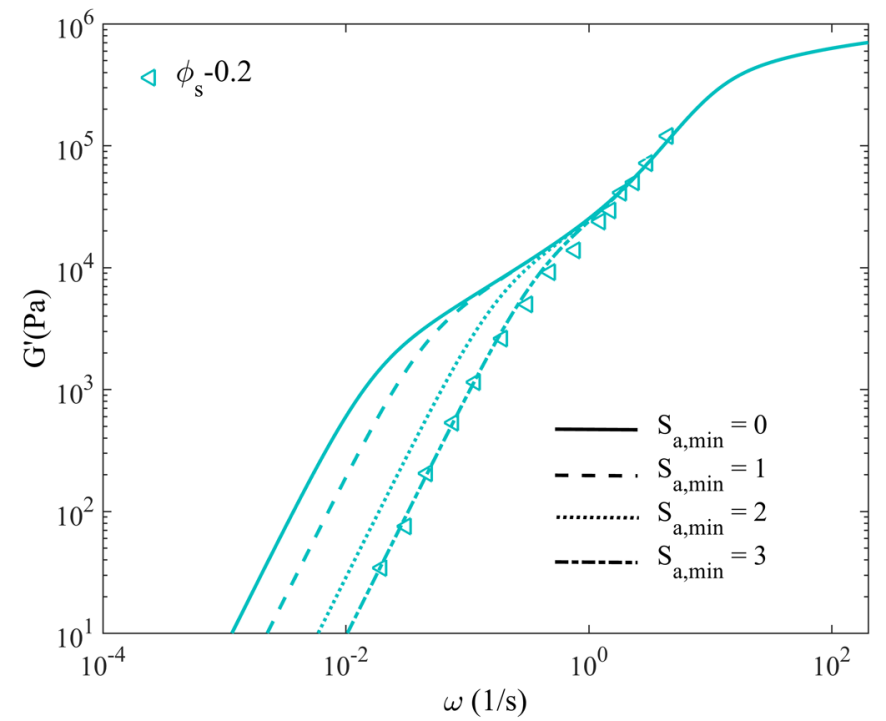

Figure A10. Storage modulus, G' for 1,4-PBd 42.5KS-105KL blends (data of Struglinski et al. cited in the Milner-McLeish paper, Ref. 5) at a star volume fraction, $\phi_{s}=0.2$. Lines are Hierarchical tube model predictions using the 'Park' input parameters and assuming arm retraction in 'thin' tube in the CR-Rouse regime and different disentanglement relaxation thresholds. Solid lines show the prediction when disentanglement relaxation process was not activated, dashed lines show disentanglement relaxation with entanglement threshold of $S_{a, \min }=$ 1, dotted lines show results for an entanglement threshold of $S_{a, \min }=2$ and dashed-dotted lines for an entanglement threshold of $S_{a, \min }=3$.

2.iii. Calculations using different algorithms within the tube model framework: Hierarchical model, BoB model and the special star-linear algorithm model developed by Park and Larson ${ }^{57}$ We also employed the BoB model to predict the rheology of 1,4-PBd 42.5KS-105KL blends in comparison to the Hierarchical model predictions as shown in Figure A11. The BoB 
model is unable to predict the rheology of pure star and pure linear component correctly as is also true of Hierarchical counter part shown in Figure A11-A. Again, the BoB model gives poor predictions for dilute star fraction blends with star fractions $\phi_{s} \leq 0.5$ because of the wide and artificially prolonged supertube relaxation region which over-estimates the modulus as compared to the experimental data. These results are qualitatively very similar to those shown in the Hierarchical model predictions for the same cases in Figure A11-A. Quantitatively, the discrepancies between the two models might be due to the differences in tube model parameters or slight differences in the way the algorithms handle numerical implementation of the different mechanisms. 

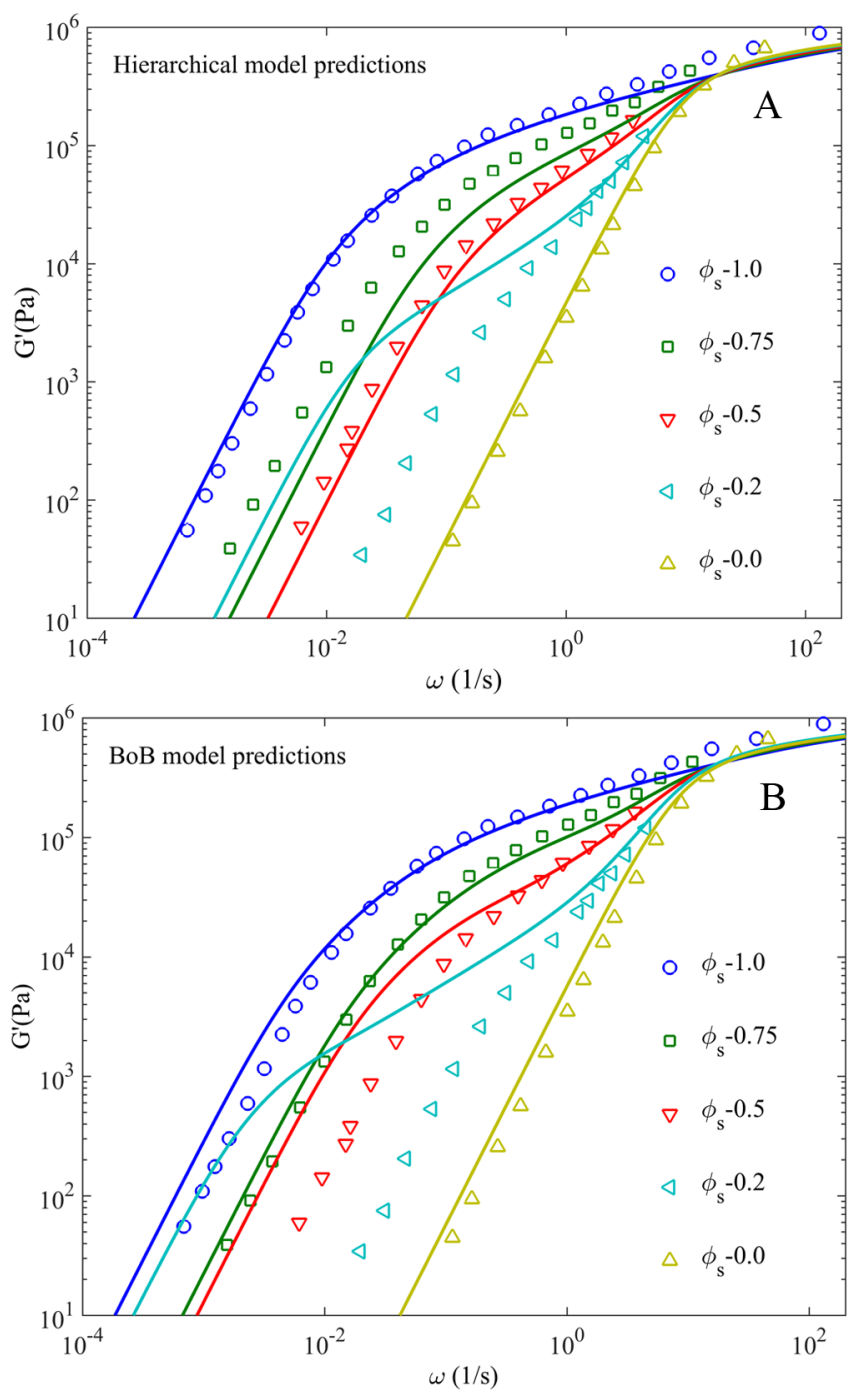

Figure A11. Storage moduli, G' for 1,4-PBd 42.5KS-105KL blends (data of Struglinski et al. cited in the Milner-McLeish paper, Ref. 5) at five different star volume fractions as shown. The symbols are experimental data at $\mathrm{T}=25^{\circ} \mathrm{C}$. In $\mathrm{A}$, solid lines are Hierarchical tube model predictions and in B, solid lines are BoB tube model predictions both obtained using the 'Park' input parameters and assuming arm retraction in 'thin' tube in the CR-Rouse regime.

Figures A12 and A13 show predictions of the special star-linear algorithm for 1,4-PBd 42.5KS-105KL blends at three different star volume fractions as shown. Again, to get the model 
agree to the experimental data for the dilute star fraction $\phi_{s}=0.2$ case, we had to include disentanglement relaxation at a threshold of $S_{a, \min }=2$ for both the Park and Park $(\alpha=1)$ input parameters as shown in Figure A12 and Figure A13, respectively. In general for this blend, Park ( $\alpha=1$ ) parameters without including disentanglement relaxation seem to work better for the other two blend cases as shown in Figure A13.

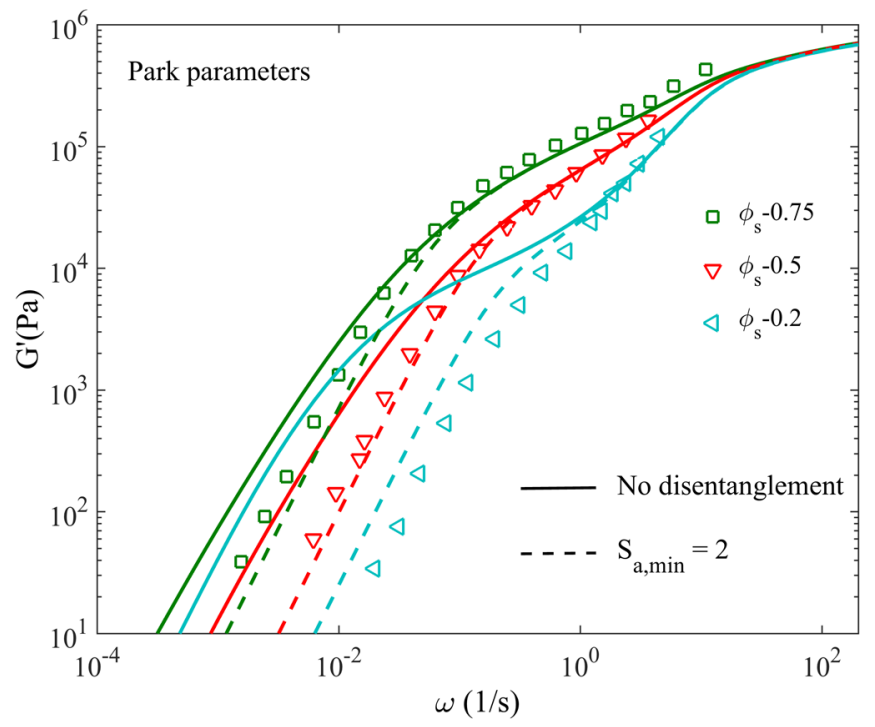

Figure A12: Storage moduli, G' for 1,4-PBd 42.5KS-105KL blends at three different star volume fractions as shown. The predictions are for the special star-linear algorithm model using "Park" input parameters from Park and $\operatorname{Larson}^{57}$. Solid lines show predictions without the disentanglement process activated. Dashed lines give predictions with disentanglement relaxation process activated at the entanglement threshold value of $S_{a, \min }=2$. 


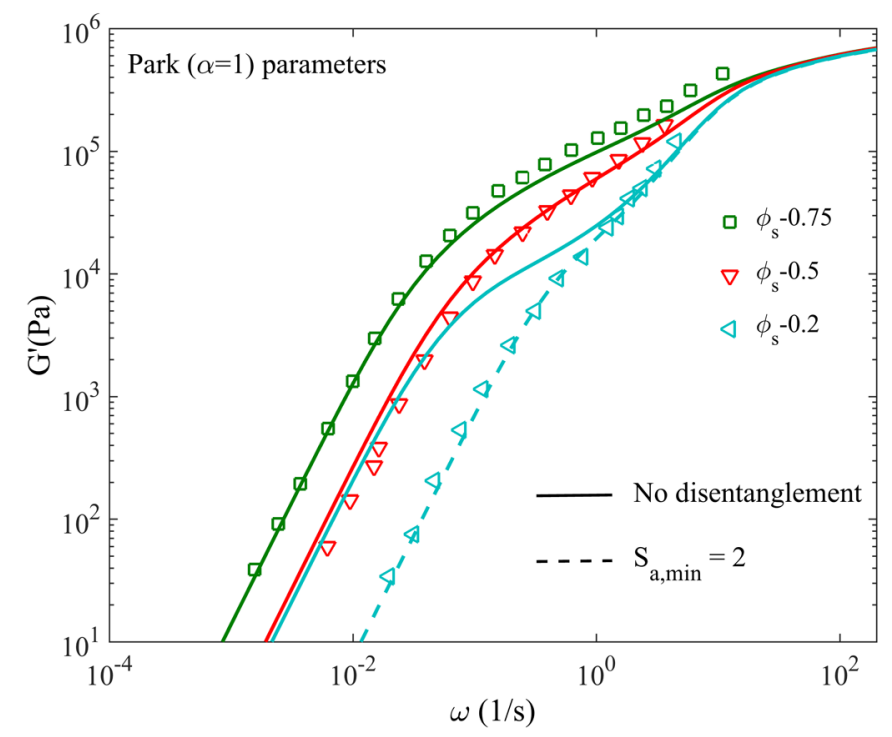

Figure A13: Storage moduli, G' for 1,4-PBd 42.5KS-105KL blends at three different star volume fractions as shown. The predictions are for the special star-linear algorithm model from Park and Larson $^{57}$ using "Park $(\alpha=1)$ " input parameters. Solid lines show predictions without the disentanglement process activated. Dashed lines give predictions with disentanglement relaxation process activated at the entanglement threshold value of $S_{a, \text { min }}=2$.

\section{Different versions of tube model predictions for data set from Shivokhin et al. ${ }^{14}(1,4-P B d$} 27.4KS-6.9KL)

3.i. Calculations using different parameter sets within the tube model: Park versus Das parameters

Figure A14 shows Hierarchical tube model predictions using both Park and Das input parameters and arm retraction in thin tube during the CR-Rouse regime, for the 1,4-PBD bidisperse blend of nominally a $24.5 \mathrm{~K}$ star blended with low molar mass linear $6.5 \mathrm{~K}$, from Shivokhin et al. ${ }^{14}$ Note that both the Park and Das parameter sets closely follow the experimental data and give very good predictions without having the need to include disentanglement 
relaxation even for the dilute star fraction cases with $\phi_{s} \leq 0.2$. In the main text (Fig. 7), we show that inclusion of disentanglement relaxation ruins these predictions for this set of blends. This contrasts with the results for our samples, $24 \mathrm{KS}-58 \mathrm{KL}$, where predictions are poor without disentanglement, but more reasonable with disentanglement with $S_{a, \min }=2$.

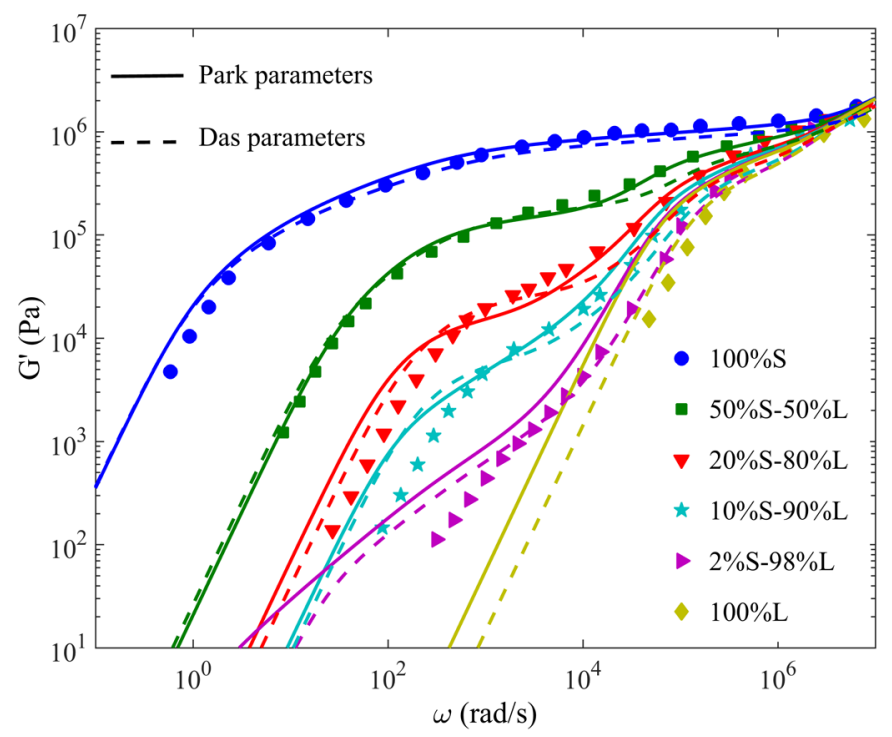

Figure A14. Storage moduli, G' for 1,4-PBd 27.4KS-6.9KL blends (data from Shivokhin et $\left.a l{ }^{14}\right)$ at six different star volume fractions as shown. The symbols are experimental data at $\mathrm{T}=25^{\circ} \mathrm{C}$. Solid and dashed lines show Hierarchical tube model predictions using Park and Das input parameters, respectively. Disentanglement relaxation was not activated for these predictions.

3.ii. Calculations using different algorithms within the tube model framework: Hierarchical model and the special star-linear algorithm model developed by Park and Larson ${ }^{57}$

We also employed the special star-linear algorithm by Park and Larson ${ }^{57}$ to predict the rheology of 1,4-PBd 27.4KS-6.9KL blends using Park parameters as shown in Figure A15, both 
with and without disentanglement relaxation. As shown, the algorithm is unable to predict the rheology of any of the blends correctly. Again, as seen with its Hierarchical predictions, switching ON disentanglement relaxation worsens the predictions for all of the cases studied.

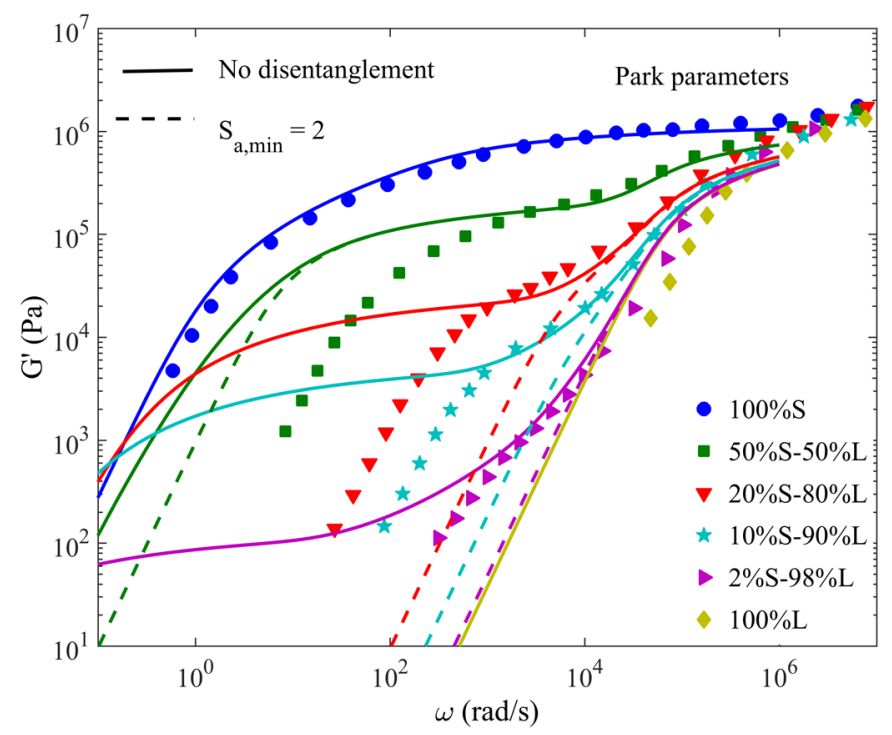

Figure A15. Same as Figure A14 except, the predictions are for the special star-linear algorithm model from Park and Larson ${ }^{57}$. Solid lines show predictions without the disentanglement process activated, while dashed lines give predictions with disentanglement relaxation process activated at the entanglement threshold value of $S_{a, \min }=2$, both using Park parameters. 


\section{Different versions of tube model predictions for data set from $\operatorname{Roovers}^{16}(1,4-P B d$}

\section{KS-23.6KL)}

\section{4.i. Calculations including the disentanglement relaxation mechanism}

Because of the arbitrariness seen with disentanglement relaxation mechanism in all of the blends studied here, it is worth seeing how well introduction of entanglement relaxation works for the Roovers ${ }^{16}$ star/linear blend case where a dilute $(2.5 \%)$ long-arm star $1,4-\mathrm{PBd}$ is mixed in a low molar mass, but entangled, linear molecule. Thus in Figure A16, we test the effect of disentanglement relaxation on this blend measured experimentally by Roovers ${ }^{58}$. For this blend, the prediction without disentanglement surprisingly gives good agreement with experimental data, while that for a disentanglement threshold of $S_{a, \min }=2$ is in disagreement with the data. This comparison shows that while use of disentanglement relaxation may give good agreement with experiments in some cases, it does not provide a robust cure for the problems of the tube model for star/linear blends.

It is important to note that for star/linear blends, whenever linear relaxation was much faster than the star arm, as was the case with data from Shivokhin et al. ${ }^{14}$ and Roovers ${ }^{16}$ we did not require disentanglement relaxation mechanism to get good predictions. For cases such as our base data set (1,4-PBd 24KS-58KL) and data set from Struglinski et al. ${ }^{13}$, where both the linear and star molecules had comparable relaxation times, we had to include disentanglement relaxation to improve the model predictions irrespective of the choice of the algorithm and input parameters. 


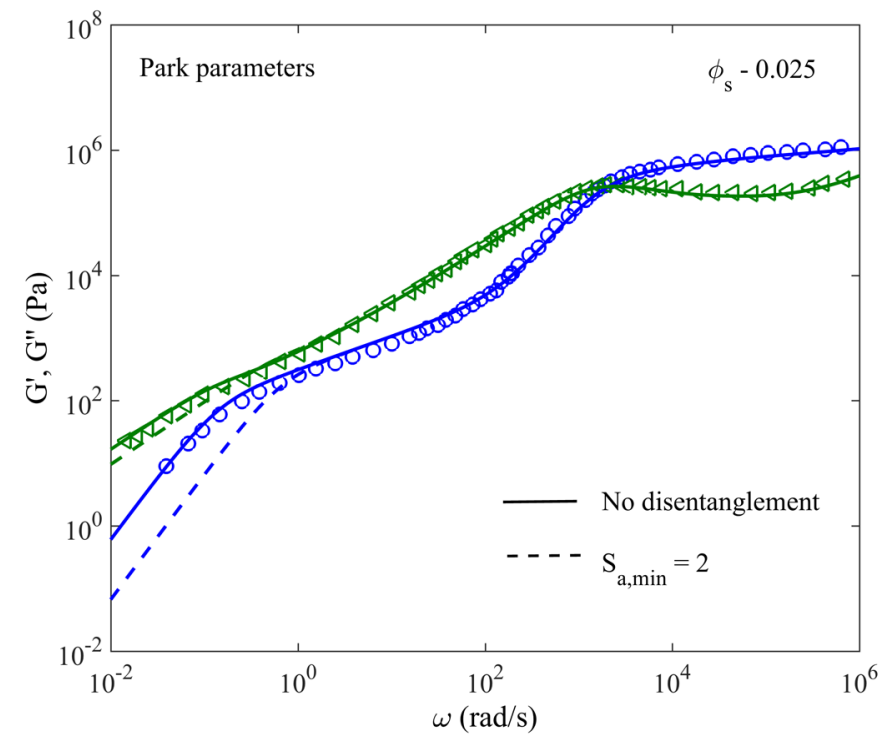

Figure A16. Comparison of Hierarchical predictions (solid lines) using Park input parameters and arm-retraction in a "thin" tube for a binary 1,4-polybutadiene blend of a nearly monodisperse linear polymer, $\mathrm{M}_{\mathrm{L}}-23,600 \mathrm{~g} / \mathrm{mol}$ with a 4-arm symmetric star of total molar mass, $\mathrm{M}_{\mathrm{S}}-1,367,000 \mathrm{~g} / \mathrm{mol}$, with arm molar mass of 342,000 at a low volume percent, $2.5 \%$ at $\mathrm{T}=27{ }^{\circ} \mathrm{C}$. Experimental data are from Roovers ${ }^{58}$. Solid lines in A represent predictions without the disentanglement relaxation process and in B represent disentanglement relaxation with a disentanglement threshold value of $S_{a, \min }=2$. 


\section{Calculations for the chain sliding dynamics (SD) and constraint dynamics (CD) contributions to modulus $G(t)$ for $1,4-P B d ~ 24 K S-58 K L$ blends}

For each of the blends of 1,4-PBd $24 \mathrm{KS}-58 \mathrm{KL}$ considered here, the corresponding contributions to $G(t)$ from SD, $G^{S D}(t)$, and CD, $G^{C D}(t)$, have been calculated using the DSM,

and plotted in Figure A17. $G^{S D}(t)$ means that only SD was turned on, and $G^{C D}(t)$ means that only the blend CD was turned on. Note that as the fraction of star-branched chains is increased, the rate of relaxation by $\mathrm{SD}$ is reduced in comparison to the rate of relaxation by $\mathrm{CD}$. The relaxation by $\mathrm{SD}$ in star chains is slowed down by the fact that entanglements can not be destroyed or created by SD at the branch point end of the arms. Entanglements can however be created and destroyed by $\mathrm{CD}$ on that end. Most of the relaxation, strand vector reorientation, happening close to the branch point occurs due to $\mathrm{CD}$. Therefore when a star chain is in a blend containing linear chains, additional relaxation can occur in this region of the chain that would not occur in a pure star system. 

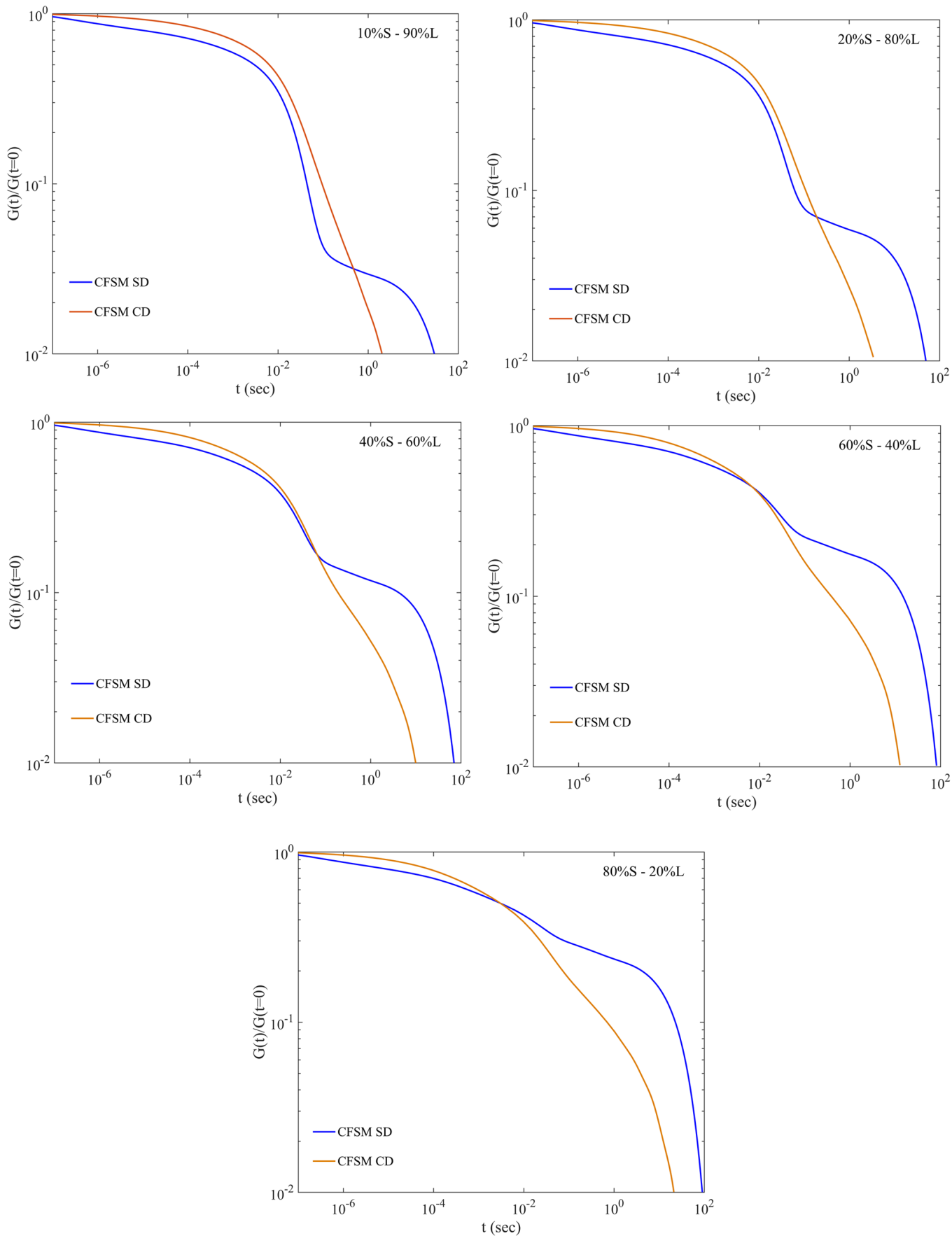

Figure A17. CFSM $G^{S D}(t)$, and $G^{C D}(t)$ predictions (lines) of PBd blends of star-branched and linear chains 


\section{Calculations using slip-spring model 1,4-PBd 24KS-58KL blends}

In this section we present results of numerical simulations conducted using Likhtman's stochastic single chain slip-spring $\operatorname{model}^{23}$. This model is based on a Rouse chain comprising $N$ beads connected by $N-1$ springs. The effect of entanglements is implemented by slip links randomly distributed along the chain. Every slip link is connected by a virtual spring with a parabolic potential to an anchoring point. Positions of the anchoring points are distributed in a way to preserve unperturbed Gaussian statistics of the chain conformations at all length scales. All implementation details and description of the model parameters can be found in Likhtman ${ }^{23}$, and Ramirez et al. ${ }^{60}$

For simulations of the stress relaxation the first step is to fit elementary bead time, $\tau_{0}$, the plateau modulus $G_{0}$ and unit mass parameter, $M_{0}$, represented by one bead in slip-spring model for all monodisperse components. Next, all binary mixtures are predicted without additional parameter fitting. Because in the earlier work, Shivokhin et al. reported molecular weights of $25.4 \mathrm{k}$ for the star arm and $7.5 \mathrm{k}$ for the linear polymer, Shivokhin et al. used a different value of $\mathrm{M}_{0}$ than is used here. Given the value of $\mathrm{M}_{0}=0.38 \mathrm{k}$ used here, and the numbers of beads $\mathrm{N}=75$ for the star arm and $\mathrm{N}=18$ for the linear chain, we re-assess the molecular weight of the star arm to be $75 \times 0.38 \mathrm{k}=28.5 \mathrm{k}$ and the linear to be $18 \times 0.38 \mathrm{k}=6.84 \mathrm{k}$. These are close to the values of $27.4 \mathrm{k}$ and $6.9 \mathrm{k}$ that we use for these materials in all our other fits. Because the parameters $\tau_{0}$, the plateau modulus $G_{0}$ were previously obtained for the $1,4-\mathrm{PBd} 27.4 \mathrm{KS}-6.9 \mathrm{KL}^{14}$, for consistency we use those same parameters for the new blends 1,4-PBd 24KS-58KL.

The slip-spring simulation results are found in dimensionless units, with thermal energy $k_{B} T=1$, statistical segment $b=1$ and friction coefficient of one segment $\zeta_{0}=1$. Regardless of molecular topology and molar mass we use $N_{e}=4$ as the average number of beads between two 
slip links, $N_{s}=0.5$ as virtual spring strength, and a unit discrete slip link jump of size 1 at every time step $d t=0.05$ in a Metropolis Monte-Carlo simulation as described in Likhtman ${ }^{59}$. For mapping the time and stress of the model to those of the experimental data, we multiply them by $\tau_{0}$ and $G_{0}$, respectively.

In this work we use a variant of the slip-spring model which imposes the condition that the monomer distance between neighboring slip links must be larger than unity (in units of the statistical segment length) thereby preventing two slip links from overlapping or occupying neighboring beads just as in Shivokhin et al. ${ }^{14}$
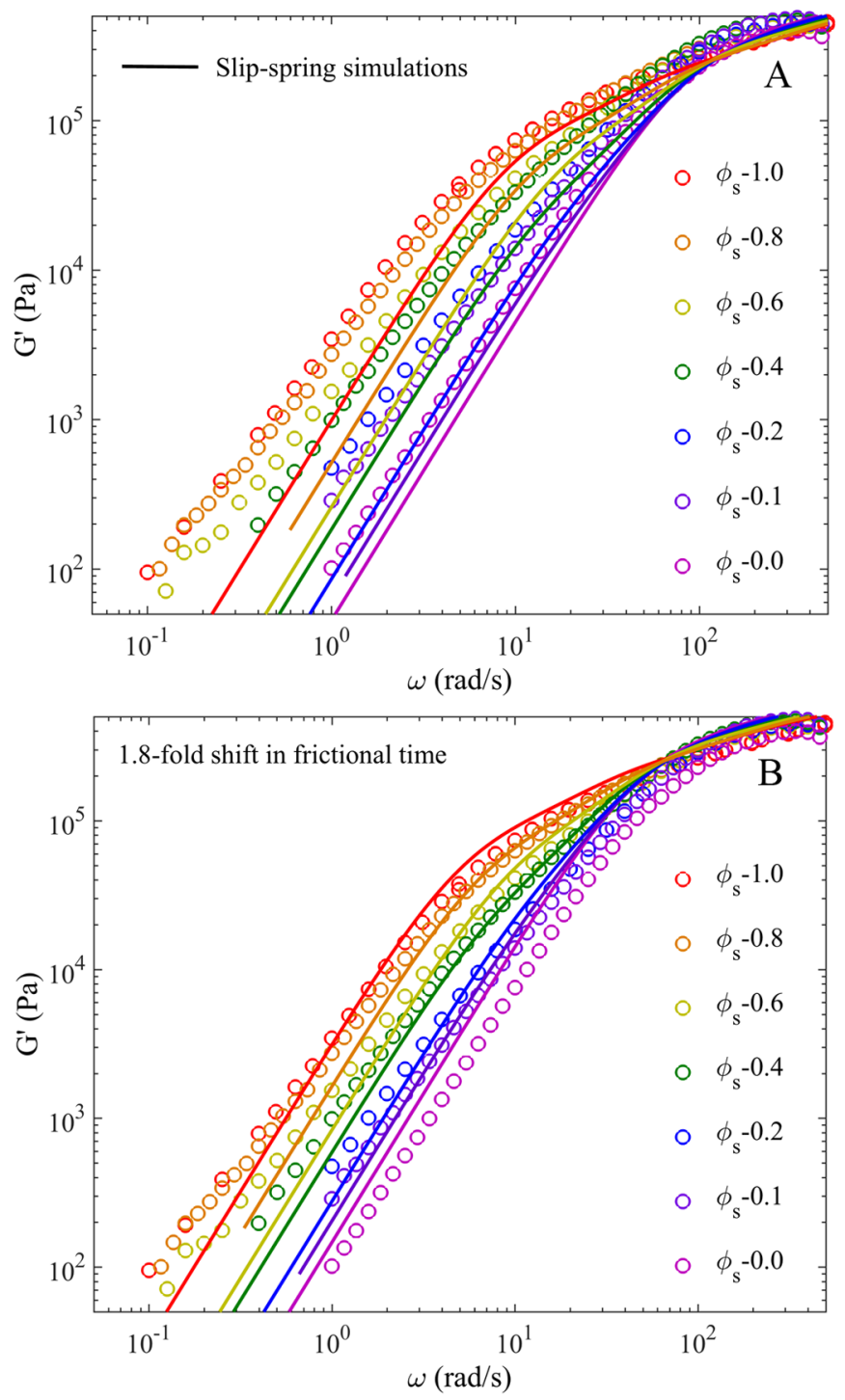
Figure A18. Storage moduli, G' for 1,4-PBd 24KS-58KL blends with decreasing fraction of star-branched chains from left to right as shown. The symbols are experimental data at $\mathrm{T}=25^{\circ} \mathrm{C}$. In both $\mathrm{A}$ and $\mathrm{B}$, solid lines show slip-spring simulations with model parameters: $M_{0}=0.38 \mathrm{kDa}$ with $\mathrm{N}=63$ beads for the star polymer (giving a molar mass of $\mathrm{N} \mathrm{x} M_{0}=24 \mathrm{~kg} / \mathrm{mol} / \mathrm{arm}$ ) and $M_{0}=0.40 \mathrm{~kg} / \mathrm{mol}$ with $\mathrm{N}=145$ beads for the linear polymer (giving a molar mass of $\mathrm{N}$ x $M_{0}=58 \mathrm{~kg} / \mathrm{mol}$ ) with $G_{0}=6.97 \mathrm{MPa}$. In A, we use with $\tau_{0}=0.25 \mu \mathrm{s}$, so that the two parameters $G_{0}$ and $\tau_{0}$ are identical to those used in the calculations of Shivokhin et al. ${ }^{14}$ (the values reported in that paper, namely $G_{0}=7.5 \mathrm{MPa}$ and $\tau_{0}=5.13 \mu \mathrm{s}$, are incorrect). In B, we shift the curves by a factor of 1.8 , so that $\tau_{0}=0.45 \mu$ s. This shift was carried out to account for the difference in high frequency rheology between the two star polymers shown in Fig. 9 in the main text.

Figure A18-A shows storage moduli, G' predictions for 1,4-PBd 24KS-58KL using slipspring simulations for 1,4-PBd 24KS-58KL blends, with parameters chosen to be the same as those used in the earlier work with 1,4-PBd 27.4KS-6.9KL. Figure A18-B shows the same slipspring predictions in Figure A18-A but with a 1.8-fold increase in the frictional time. This shift was done to account for the slower high-frequency relaxation of the $24 \mathrm{~K}$ star as compared to the 27.4K Shivokhin star (see Figure 9 in main text), since the latter was used to obtain the fitting parameters. Note that the slip-spring model gives a reasonable, but not perfect, prediction of the data. Either the pure star or the pure linear material is incorrectly predicted in Fig. A18A\&B.

It should be noted that the stress of the model is calculated in units of $G_{0}=\rho R T / M_{0}$ with $\rho$, $R$ and $T$ being the polymer density, universal gas constant and absolute temperature, respectively ${ }^{23}$. By using $\rho(\mathrm{PBd})=950 \mathrm{~kg} \cdot \mathrm{m}^{-3}, \mathrm{~T}(\mathrm{PBd})=298 \mathrm{~K}, \mathrm{R}=8.31 \mathrm{~m}^{3} \cdot \mathrm{Pa} \cdot \mathrm{K}^{-1} \cdot \mathrm{mol}^{-1}$ and by substituting the fitted value of $M_{0}$ we compute $G_{0}(\mathrm{PBd})=5.9 \mathrm{MPa}$. This value is different from 
the fitted $G_{0}$ by $\sim 15 \%$, which is within variations of data reported in different papers. The model stress $G_{0}$ should not be confused with the plateau modulus $G_{N}{ }^{0}$. 